$\mathbb{T}$ periodica polytechnica

\author{
Transportation Engineering \\ $40 / 1(2012) 2732$ \\ doi: 10.3311/pp.tr.2012-1.05 \\ web: http://www.pp.bme.hu/tr \\ (c) Periodica Polytechnica 2012
}

RESEARCH ARTICLE

\section{Active suspension control design for unmanned ground vehicles}

\author{
Dávid Nagy / Péter Gáspár
}

Received 2012-10-27

\begin{abstract}
This paper presents the design of an active suspension control system for an unmanned ground vehicle (UGV). The purpose is to design an active suspension control for a low-speed (less than $1 \mathrm{~m} / \mathrm{s}$ ) off-road UGV in order to be able to move through rugged terrain with the least pitch and roll motion. Classical active suspension design methods cannot be used for minimizing pitch and roll angles, therefore a new approach is applied. The control design is based on the LQG method. The control system uses only pitch and roll angular rate signals, which ensures a simple and cheap control system, but any bias error on the gyro signals cause some problems in reconstructing angles. The control algorithm consists of an optimal state-feedback fed by an augmented observer for estimating the states and the bias error of the gyro sensors. The appropriate tuning of the observer is introduced, which eliminates the bias error problem and ensures the fast reconstruction of the states for the optimal state-feedback. In simulations, the active suspension control system shows high performance at minimizing pitch and roll angles.
\end{abstract}

\section{Keywords}

LQG control design · model building · active suspension . $U G V \cdot$ sensor bias error estimation

\section{Acknowledgement}

This work was supported by the Hungarian National Office for Research and Technology through grant TECH_08_2/22008-0088 and the Hungarian National Science Foundation (OTKA) through grant CNK-78168 which are gratefully acknowledged.

\section{Dávid Nagy}

Department of Control for Transportation and Vehicle Systems, BME, H-1111 Budapest, Stoczek J. u., Hungary

e-mail: nagydav@sztaki.hu

\section{Péter Gáspár}

Systems and Control Laboratory, Computer and Automation Research Institute,MTA, Kende u. 13-17, H-1111 Budapest, Hungary

e-mail: gaspar@sztaki.hu

\section{Introduction}

Interest in unmanned ground vehicles (UGVs) has been steadily growing. UGVs are spreading in search-and-rescue and military applications as well as in civil or research projects. The intensive development of electrical and digital computing sciences has produced high-performance, reliable and cheap devices, which enable the implementation of complex control systems for autonomous vehicles. UGVs, however, pose numerous control problems, see e.g. [2 10]

One of the main parts of the autonomous vehicle is the active suspension system because it predetermines the motions of the vehicle body. This paper summarizes the design process of an active suspension control system for a low-speed (less than $1 \mathrm{~m} / \mathrm{s}$ ) UGV. Several methods have been proposed to solve the automotive active suspension problem, [1, 4,6, 8]. Automotive active suspension control systems focus on road-holding, comfort and stability during manoeuvres. In contrast, a robotic offroad vehicle can move slowly, the excitation is rugged terrain instead of a road, and comfort is unimportant. The objective of the active suspension control of this UGV is to keep the pitch and roll angles of the chassis in the neighbourhood of zero, i.e., to minimize the absolute of these angles. Keeping the vehicle body in horizontal position is a relevant factor in case of a very rough terrain, or when carrying a casualty or dangerous material. Measuring pitch and roll angles could be ideal for control system but direct angle measurement during travel is complicated or requires expensive sensors. Classical methods of active suspension control design cannot be used for minimizing pitch and roll angles. Therefore a new approach is applied.

The base of this robotic vehicle is a commercial small-scale vehicle because it contains the main mechanical parts. Four hobby servo motors are applied for actuating the four individual suspensions. A full-car vehicle model and the LQG method are used for control design. The control system uses pitch and roll rate signals measured by a six-degree-of-freedom inertial sensor unit with three accelerometers and three gyro sensors. Using only angular rate sensors is a simple and cheap solution but poses some problems. On the one hand, it is necessary to reconstruct the states for the optimal state-feedback with an observer. 
On the other hand, due to a kind of integration operation at the reconstruction of the angle states from angular rates, any bias error on the angular rate signals causes an endless increase (or decrease) on the observed angle states. Therefore the observer has to estimate sensor bias errors as well. The parameters of the control algorithm have been tuned by MATLAB/Simulink simulations. These simulations show that the problem of the bias errors can be eliminated, furthermore the active suspension vehicle is able to move with much less pitch and roll motion than the similar but passive suspension vehicle.

The structure of the paper is as follows. Section 2 introduces the system model, then Section 3 details the control design process considering the implementation problems. The results of the simulations are discussed in Section 4.

\section{System model and dynamics}

The goal of the control design is to reduce the pitch and the roll motion of the UGV chassis with using only pitch and roll rate sensors. For handling these motions a full-vehicle model is required. The base of the UGV has four mechanically independent suspensions. The vehicle has to be able to move through rugged terrain, thus the suspension system must enable high suspension deflection. Therefore the concept is that servo motor actuator joins serial with the original spring-damper part, that means servo is able to actuate the upper joint of the spring-damper element. This concept is advantageous because a high wheel displacement can be continuously ensured with low power consumption, in contrast to such systems, in which actuators and passive elements are parallel. Using passive elements are necessary for ensuring correct wheel-ground contact in case of high frequency excitation or even actuator failure. This concept is detailed in [9].

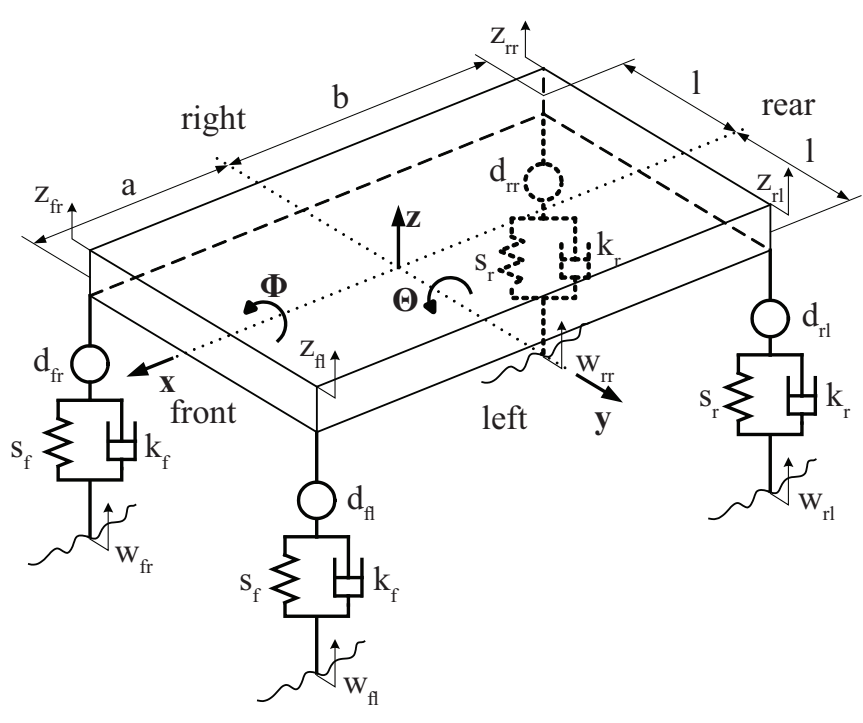

Fig. 1. Model of full-vehicle system
A control-oriented system model is built for control design. The model is shown in Fig. 1. The full-vehicle suspension system is represented as a linearised three-degree-of-freedom (DOF) system. The sprung mass (with mass $m$ and moment of inertia around $x$-axis and $y$-axis $J_{x}$ and $\left.J_{y}\right)$ is free to heave $(z)$, pitch $(\theta)$ and roll $(\phi)$. For simplicity, tires are neglected because it is assumed that wheels are able to track the surface with negligible vibration at low speed. Therefore the terrain excitation $\left(w_{f r}, w_{f l}, w_{r r}, w_{r l}\right)$ is transmitted to the sprung mass by the spring-damper parts and the actuators. The passive elements are modelled as linear viscous dampers (with damping coefficients $k_{f}$ and $k_{r}$ ) and linear springs (with stiffness $s_{f}$ and $s_{r}$ ). Each actuator realizes a defined displacement $\left(d_{f r}, d_{f l}, d_{r r}, d_{r l}\right)$ between the corner of the sprung mass and the upper joint of the spring-damper element. Center of Gravity ( $\mathrm{CoG})$ co-ordinate system is used for motion equations; the concept of [7] is followed. The origin is at the vehicle center-of-gravity and the coordinate system moves with the vehicle during travel. Trigonometric relationship, between the body corner displacements $\left(z_{f r}\right.$, $\left.z_{f l}, z_{r r}, z_{r l}\right)$ and the heave $(z), \operatorname{pitch}(\theta)$ and roll $(\phi)$ motions of the sprung mass can be linearised because the control system keeps the pitch and roll angles in the neighbourhood of zero.

The matrix equation of the motions are

$$
M \ddot{q}=K \dot{q}+S q+B_{k} \dot{w}+B_{s} w+B_{k} \dot{d}+B_{s} d
$$

where the vector variables are

$$
q=\left[\begin{array}{l}
z \\
\theta \\
\phi
\end{array}\right], \quad w=\left[\begin{array}{l}
w_{f r} \\
w_{f l} \\
w_{r r} \\
w_{r l}
\end{array}\right], \quad d=\left[\begin{array}{l}
d_{f r} \\
d_{f l} \\
d_{r r} \\
d_{r l}
\end{array}\right]
$$

and the constant matrices are

$$
\begin{aligned}
& K=\left[\begin{array}{ccc}
-2\left(k_{f}+k_{r}\right) & 2\left(k_{f} a-k_{r} b\right) & 0 \\
2\left(k_{f} a-k_{r} b\right) & -2\left(k_{f} a^{2}+k_{r} b^{2}\right) & 0 \\
0 & 0 & -2 l^{2}\left(k_{f}+k_{r}\right)
\end{array}\right] \\
& S=\left[\begin{array}{ccc}
-2\left(s_{f}+s_{r}\right) & 2\left(s_{f} a-s_{r} b\right) & 0 \\
2\left(s_{f} a-s_{r} b\right) & -2\left(s_{f} a^{2}+s_{r} b^{2}\right) & 0 \\
0 & 0 & -2 l^{2}\left(s_{f}+s_{r}\right)
\end{array}\right] \\
& M=\left[\begin{array}{ccc}
m & 0 & 0 \\
0 & J_{y} & 0 \\
0 & 0 & J_{x}
\end{array}\right], B_{k}=\left[\begin{array}{cccc}
k_{f} & k_{f} & k_{r} & k_{r} \\
-a k_{f} & -a k_{f} & b k_{r} & b k_{r} \\
-l k_{f} & l k_{f} & -l k_{r} & l k_{r}
\end{array}\right] \\
& B_{s}=\left[\begin{array}{cccc}
s_{f} & s_{f} & s_{r} & s_{r} \\
-a s_{f} & -a s_{f} & b s_{r} & b s_{r} \\
-l s_{f} & l s_{f} & -l s_{r} & l s_{r}
\end{array}\right]
\end{aligned}
$$

The actuator (servo motor) also has dynamics which cause some delay at the realization of the control input signals. The installed commercial servo motors include control systems. An abstract model of the complete servo actuator is a system which has an input of the reference position and an output of the actual position. A first-order linear system model is applied in this 
control-oriented model because it is simple but accurate enough to describe the main characters of the actuator. Therefore, actuator dynamics is modelled with the following differential equation

$$
\dot{d}_{i}=-\frac{1}{T_{d}} d_{i}+\frac{A_{d}}{T_{d}} u_{i}
$$

where $u_{i}$ denotes one of the control input signal and the corresponding actuator state is denoted by $d_{i}$; the indexes $i$ can be $f r$, $f l, r r$ or $r l . T_{d}$ and $A_{d}$ are the time and amplification constants. The state of the unsprung mass can be described by the three position (angle) and three velocity (angular rate) coordinates: heave, pitch, roll positions and the corresponding derivatives. Due to the dynamics of the actuators, these states must be augmented by the four actuator positions for describing the complex system. The system equation in state-space representation is

$$
\dot{x}=A x+B u+B_{1} \dot{w}+B_{2} w
$$

where the state vector is

$$
x=\left[\begin{array}{llllllllll}
\dot{z} & \dot{\theta} & \dot{\phi} & z & \theta & \phi & d_{f r} & d_{f l} & d_{r r} & d_{r l}
\end{array}\right]^{T}
$$

and the input vector is

$$
u=\left[\begin{array}{llll}
u_{f r} & u_{f l} & u_{r r} & u_{r l}
\end{array}\right]^{T} .
$$

The constant matrices are (where $I$ and $O$ denote the appropriate size unit and zero matrix blocks)

$$
\begin{gathered}
A=\left[\begin{array}{ccc}
M^{-1} K & M^{-1} S & M^{-1} B_{k}\left(-\frac{1}{T}\right) I+M^{-1} B_{s} \\
I_{3 \times 3} & O_{3 \times 3} & O_{3 \times 4} \\
O_{4 \times 3} & O_{4 \times 3} & \left(-\frac{1}{T}\right) I_{4 \times 4}
\end{array}\right] \\
B=\left[\begin{array}{c}
M^{-1} B_{k}\left(\frac{A}{T}\right) I \\
O_{3 \times 4} \\
\left(\frac{A}{T}\right) I_{4 \times 4}
\end{array}\right], B_{1}=\left[\begin{array}{c}
M^{-1} B_{k} \\
O_{7 \times 4}
\end{array}\right], B_{2}=\left[\begin{array}{c}
M^{-1} B_{s} \\
O_{7 \times 4}
\end{array}\right]
\end{gathered}
$$

\section{Design of output-feedback controller}

The control algorithm consists of an optimal state feedback and an optimal observer. According to the separation principle, the optimal state feedback and the observer are designed independently. Classical output feedback structure is followed (see Fig. 2), i.e., an optimal observer reconstructs the states denoted by $x$, and the estimated state vector $x_{k}$ feeds into an optimal controller ( $-K$ feedback).

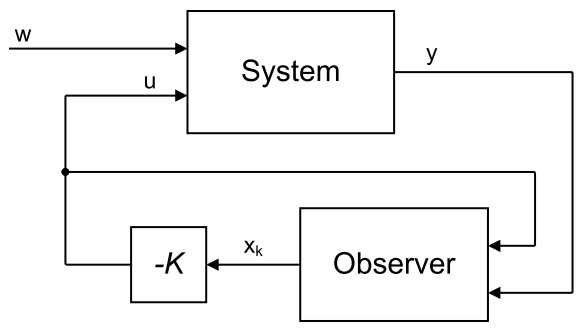

Fig. 2. Structure of the output-feedback system
The objective of the control is to minimize the pitch and the roll angles of the UGV chassis. The optimal state feedback is calculated by the linear quadratic method. Therefore the following objective function has to be minimized:

$$
J=\int_{0}^{\infty}\left\{q_{\theta} \theta^{2}+q_{\phi} \phi^{2}+r u_{f r}+r u_{f l}+r u_{r r}+r u_{r l}\right\} \mathrm{d} t
$$

where $q_{\theta}$ and $q_{\phi}$ are the weights corresponding to $\theta$ and $\theta$ angles (states), and $r$ is the weight of the control signals, respectively. The weight factors has been tuned by simulations. The optimal feedback gain vector is calculated by the lqr MATLAB function.

All the states are not measurable so that an observer is required. Another problem is that pitch and roll angles are not measurable directly but only pitch and roll rates. Thus the observer does a kind of integral operation to calculate the angles from the measured angular rates. Unfortunately real gyro sensors have some noises and a temperature-dependent bias error which causes an endless increase (or decrease) of the angle states because of the integrator effect. To eliminate this problem, the observer has to estimate the sensor bias errors besides reconstructing the states from the measured signals. For this, the state vector is augmented by the two bias errors of the pitch and roll rate sensors: $x^{\prime}=\left[\begin{array}{lll}x^{T} & b_{\theta} & b_{\phi}\end{array}\right]^{T}$. The equation of the augmented system is (without the terrain excitation)

$$
\dot{x}^{\prime}=\left[\begin{array}{cc}
A & 0 \\
0 & 0
\end{array}\right] x^{\prime}+\left[\begin{array}{l}
B \\
0
\end{array}\right] u
$$

Pitch and roll angular rates and the four actuator states are measured for reconstructing the states of the vehicle. Therefore the output vector is

$$
y=\left[\begin{array}{llllll}
\dot{\theta} & \dot{\phi} & d_{f r} & d_{f l} & d_{r r} & d_{r l}
\end{array}\right]^{T}
$$

The observation equation is

$$
y=C x+b=\left[\begin{array}{ll} 
& I_{2 \times 2} \\
& O_{4 \times 2}
\end{array}\right] x^{\prime}
$$

where $b=\left[\begin{array}{llllll}b_{\theta} & b_{\phi} & 0 & 0 & 0 & 0\end{array}\right]^{T}$ and the constant matrix $C$ is

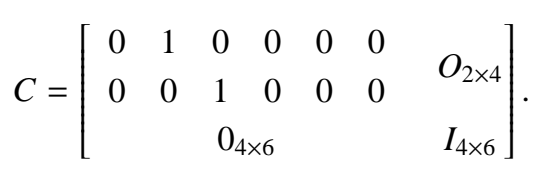

The optimal feedback of the output error is calculated by the linear quadratic method, using the dual pair of the system defined by Eqs. 5 and 6 The aim is to reconstruct the states as fast as possible and estimate the bias errors with slow dynamics. Slow dynamics is necessary to keep the performance of the optimal state-feedback in case of higher frequencies (up to 1$2 \mathrm{~Hz}$ ), otherwise all the terrain excitation are observed as bias error. The objective function is

$$
J=\int_{0}^{\infty}\left(x(t)^{T} Q_{O} x(t)+u(t)^{T} R_{O} u(t)\right) \mathrm{d} t
$$


where the weight matrices are supposed as $Q_{O}=\left\langle\begin{array}{llllllllllll}q_{1} & q_{1} & q_{1} & q_{2} & q_{2} & q_{2} & q_{3} & q_{3} & q_{3} & q_{3} & q_{4} & q_{5}\end{array}\right\rangle$ and $R_{O}=\left\langle r_{1} r_{1} r_{1} r_{1} r_{1} r_{1}\right\rangle$ diagonal matrices. These weights have been tuned by simulations. It is found these assortment of the weight factors simplified the tuning of the observer, without reducing its performance.

\section{Simulation results}

Simulations are used for tuning the state-feedback and the observer as well as analysing the controlled system. Implementation of the control system on the small-scale car is considered during the design process and simulations. The measured and identified parameters (see Table 1 ) of the developing UGV are used in simulations.

Tab. 1. Measured and identified parameters of the UGV

\begin{tabular}{cc}
\hline Parameter & Value \\
\hline Mass $(m)$ & $1.868 \mathrm{~kg}$ \\
Inertia around $y$-axis $\left(J_{y}\right)$ & $0.02581650 \mathrm{kgm}^{2}$ \\
Inertia around $x$-axis $\left(J_{x}\right)$ & $0.01072268 \mathrm{kgm}^{2}$ \\
Front spring stiffness $\left(s_{f}\right)$ & $247 \mathrm{~N} / \mathrm{m}$ \\
Rear spring stiffness $\left(s_{r}\right)$ & $134 \mathrm{~N} / \mathrm{m}$ \\
Front damping coeff. $\left(k_{f}\right)$ & $12 \mathrm{Ns} / \mathrm{m}$ \\
Rear damping coeff. $\left(k_{r}\right)$ & $15 \mathrm{Ns} / \mathrm{m}$ \\
Half wheelbase $(l)$ & $0.104 \mathrm{~m}$ \\
Distance from CoG to Front axle $(a)$ & $0.153 \mathrm{~m}$ \\
Distance from CoG to Rear axle $(b)$ & $0.121 \mathrm{~m}$ \\
Time const. of actuator model $\left(T_{d}\right)$ & $0.1254 \mathrm{~s}$ \\
Amp. const. of actuator model $\left(A_{d}\right)$ & 1 \\
\hline
\end{tabular}

First an appropriate state-feedback is determined, assuming that all the states are measurable. Fig. 3 shows the case when the front right wheel of the system is excited with a bump. The body of the active suspension vehicle has a slight pitch and roll angles during the bump excitation (solid lines) in contrast to the passive vehicle (dashed lines) which has the same passive elements. The actuators stay in their working range during this example.

The next step is to tune the observer for reconstructing the states as fast as possible. The most important states are the pitch and the roll angles because they have the major weights in the LQ criteria of the state-feedback. Fig. 4 shows the real (dashed lines) and the observed pitch and roll states (solid lines) in case of the previous bump excitation at the front right wheel (see Fig. 3/a). The diagrams of Fig. 4/a show the case where state-feedback is not applied, and output-feedback is shown by the diagrams of Fig. 4/b. The difference between the real and the observed states is negligible small in both cases (it is insensible in these figures), thus working of the observer is acceptable. The output-feedback system ensures a slight pitch and roll angles during bump test, similar to the state-feedback system.

The problem is this observer works with only ideal sensors. Without any reference angle value (angle measuring is complicated and it requires expensive sensors), angle states have a continuous increase (or decrease) in case of any bias error on the

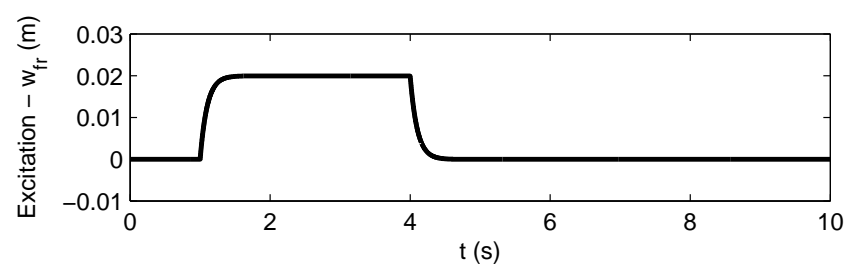

(a) Bump excitation at front right wheel
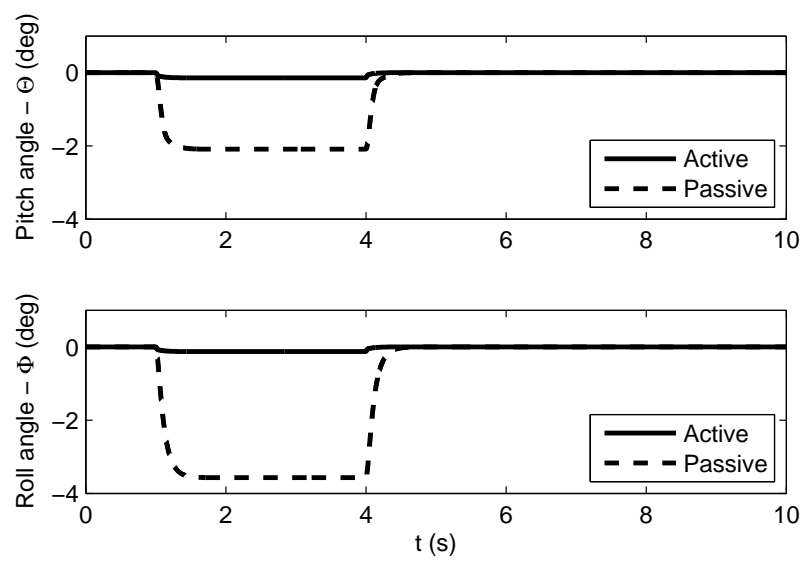

(b) Pitch and roll angle states

Fig. 3. Response of state-feeback system to bump excitation; weights are: $q_{\theta}=10^{4}, q_{\phi}=10^{4}$ and $r=10^{2}$

angular rate signals because of the integrator effect. For eliminating this problem, the observer is tuned to slow dynamics for bias error estimation. Slow dynamics ensures to keep acceptable performance while stopping the increase of the amplitude of the angle states. Fig. 5/a shows the previous bump test (excitation is shown in Fig. 3/a) with simulated bias errors on the sensor signals. (These errors are greater than the real sensor bias errors in order to emphasize the problem.) The dotted line marks the pitch and roll states of the passive vehicle. The dashed line is for the system with an observer, which does not estimate the bias errors. In this case the amplitude of the angle states are continuously increasing but the state-feedback compensates well the bump at the first section of the test. The solid line shows the output-feedback controlled system where the observer is tuned to fast reconstruction of the states and slow dynamics for the sensor bias error estimation. The performance of this system is lower than the simple observer's, but much higher than the passive system's. In case of steady state excitations the observer recognises that as bias errors. Higher frequency components (e.g. the edges of the bump) are compensated in contrast to the passive vehicle.

The working of this observer can be examined in Fig. 55/b. The amplitude of the observed pitch state (solid line in the first diagram) is lower than the real state (dotted line) because observer recognises a part of the excitation as bias error. The estimated bias error (solid line) is shown in the second diagram; the dotted line represents the simulated sensor bias error. The estimated 

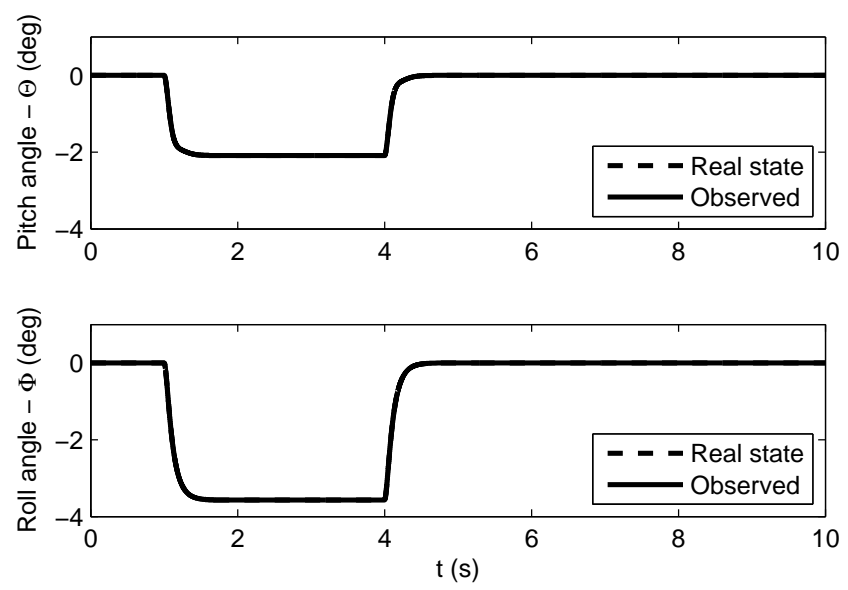

(a) Reconstruction of states without any control feedback
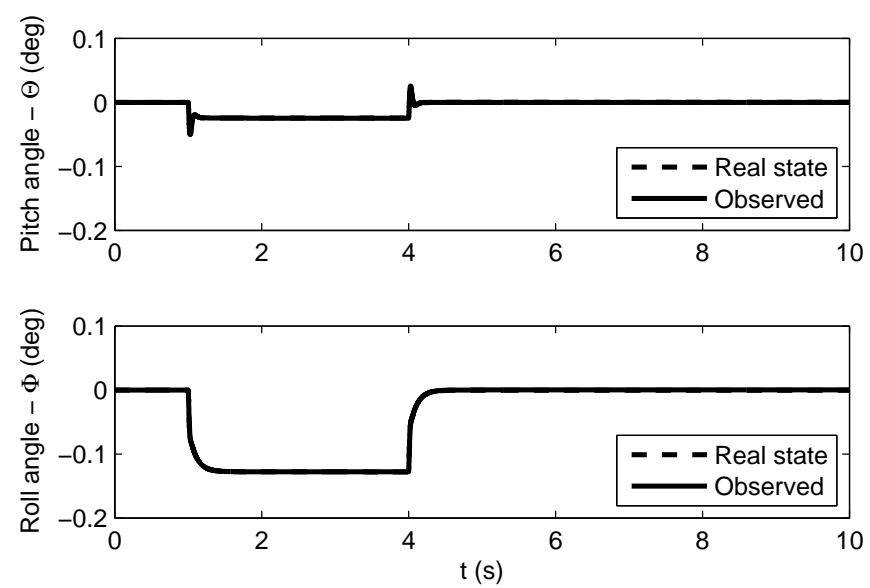

(b) Reconstruction of states with output-feedback

Fig. 4. Working of observer with low weights for sensor bias errors; weights of observer are: $q_{1}=10^{9}, q_{2}=0, q_{3}=0, q_{4}=q_{5}=10^{-15}$ and $r_{1}=10^{-5}$

error converges to the actual error in steady state. This example shows only the pitch dynamics but the roll dynamics is similar. In case of a step function excitation (non-zero steady state), the vehicle adapts slowly to the terrain by observing excitation as bias error. This problem cannot be eliminated without any reference angle measurement.

The strength of this control system is that it is able to keep the angle states in the neighbourhood of zero with only gyro sensors, in case of a zero mean oscillating terrain excitation. Fig. 6 shows a test case where the terrain excitations are zero mean oscillating signals. A modified Hac road model is used for modelling the terrain profile, [3, 5]. Simulation shows that the objective of the control system is performed: the pitch and roll states are minimized and the effects of the sensor bias errors are eliminated.
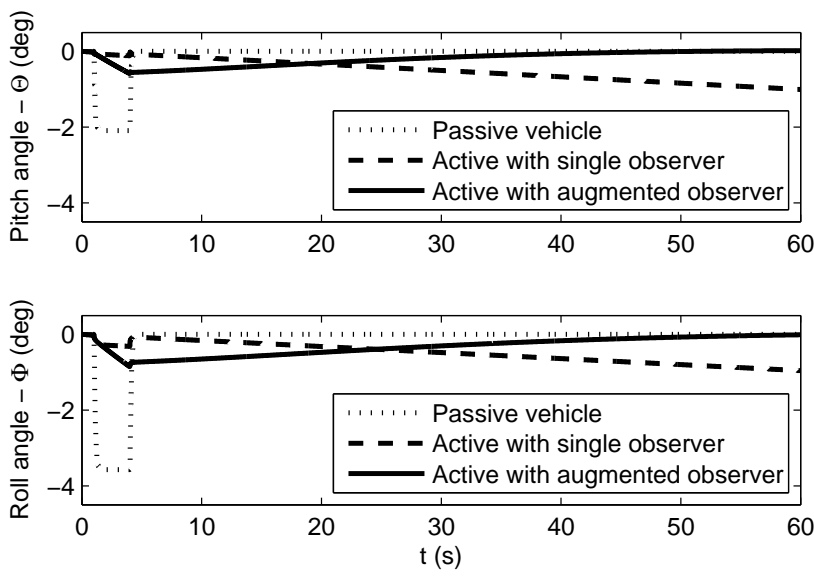

(a) Comparison of systems
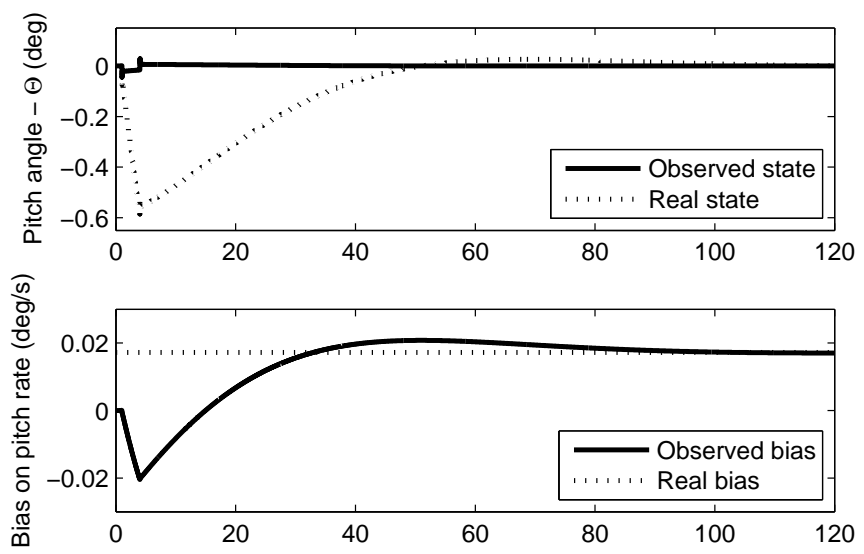

(b) Bias error estimation (Pitch angle)

Fig. 5. Bump test with simulated sensor bias errors; weights of bias errors are: $q_{4}=1$ and $q_{5}=10^{-2}$

\section{Conclusion}

This paper presents the design of an active suspension control system for an UGV. The vehicle is modelled by a threedimensional linearised full-car model for control design. The control algorithm consists of an optimal state-feedback and an optimal observer calculated by the linear quadratic method. The objective of the control system is to minimize the pitch and the roll angles of the vehicle with only two angular rate sensors. The observer does a kind of integration operation for reconstructing the angle states, which cause endless increase of the observed angles. This problem is eliminated by augmenting the observer with the two bias error states. An appropriate tuning of the observer is introduced, which ensures acceptable performance and eliminates the problem of the sensor bias errors. Simulations show that the active suspension vehicle has slight pitch and roll amplitudes during moving through rugged terrain, in contrast to the similar but passive vehicle. 

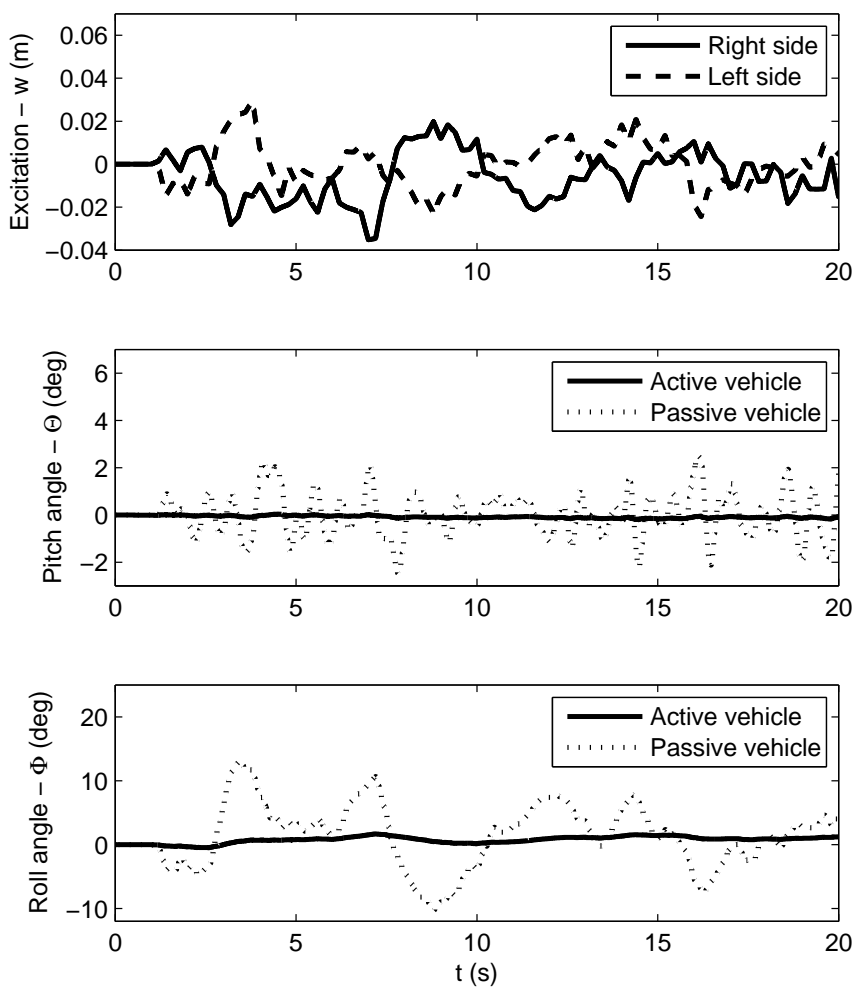

Fig. 6. Comparison of active and passive systems, with a zero mean oscillating excitation (rugged terrain)

\section{References}

1 Bokor J, Palkovics L, Michelberger P, Várlaki P, Design of active wheel suspension system using RLQR and $\mathcal{H}_{\infty}$ control, Proc. of the 12th IFAC World Congress, Sydney, 1993.

2 Feng J, Zheng S, Yu: F, Bandwidth-limited active suspension controller for an off-road vehicle based on co-simulation technology 3 (2008), 111-117, DOI 10.1007/s11465-008-0020-7.

3 Gáspár P, Szabó Z, Bokor J, Parameter identification of a suspension system and road disturbance estimation, International Journal for Vehicle Systems Modeling and Testing 2 (2007), 128-137, DOI 10.1504/IJVSMT.2007.013891.

4 Guglielmino E, Sireteanu T, Stammers C W, Ghita G, Giuclea M, Semiactive Suspension Control, Springer, 2008.

5 Hac A, Adaptive control of vehicle suspension, Vehicle System Dynamics 16 ( 1987), 57-74, DOI 10.1080/00423118708968870.

6 Hrovat D, Survey of advanced suspension developments and related optimal control applications, Automatica 33 ( 1997), 1781-1817, DOI 10.1016/S0005-1098(97)00101-5.

7 Kiencke U, Nielsen L, Automotive control systems for engine, driveline and vehicle, Springer, Berlin, 2005. isbn= 978-3-540-26484-2.

8 Michelberger P, Bokor J, A. Keresztes A, Várlaki P, Design of active suspension system for road vehicles: An eigenstructure assignment approach, Proc. of the 23th Fisita Congress (1990), 213-218.

9 Nagy D, Design of an autonomous off-road vehicle, 2010. TDK report, BME.

10 Waldron K J, Abdallah M E, An Optimal Traction Control Scheme for Off-Road Operation of Robotic Vehicles, IEEE/ASME Transactions on Mechatronics 12 (April 2007), no. 2, 1083-4435, DOI 10.1109/TMECH.2007.892819. 